\title{
Quantitative phosphoproteomics reveals a cluster of tyrosine kinases that mediates Src invasive activity in advanced colon carcinoma cells.
}

Cédric Leroy ${ }^{1}$, Camille Fialin ${ }^{1}$, Audrey Sirvent ${ }^{1}$, Valérie Simon ${ }^{1}$, Serge Urbach ${ }^{2}$, Joël Poncet ${ }^{2}$, Bruno Robert ${ }^{3}$, Patrick Jouin ${ }^{2} \&$ Serge Roche ${ }^{1}$

${ }^{1}$ CNRS UMR5237, University of Montpellier 1 and 2, CRBM, 34000 Montpellier, France

${ }^{2}$ UMR 5203 INSERM U661, University of Montpellier 1 and 2, IGF, 34000 Montpellier, France

${ }^{3}$ INSERM U896, IRCM, CRCL Val d'Aurelle Paul Lamarque, University of Montpellier 1 and 2, 34000 Montpellier, France

Correspondence: Serge Roche CRBM, UMR5237, 1919 route de Mende

34293 Montpellier Cedex 05, France.

Tel: +33467613373

Fax: 33467521559

e-mail: Serge.Roche@crbm.cnrs.fr

Running title: Src oncogenic signaling in colon carcinoma cells

Abbreviations: tyrosine kinase Src, oncogenic signaling, colorectal cancer, phosphoproteomics, invasion 


\section{ABSTRACT}

The non-receptor tyrosine kinase Src is frequently overexpressed and/or activated in human colon carcinoma (CRC) and its increased activity has been associated with a poor clinical outcome. Src has been then implicated in growth and invasion of these cancer cells by still not well known mechanisms. Here we addressed these Src oncogenic signaling using quantitative phosphoproteomics. Src overexpression increased growth and invasiveness of the metastatic SW620 CRC cells. Stable Isotope Labeling with Amino Acids in Cell Culture (SILAC) in combination with LC-MS/MS allowed the identification of 136 proteins which exhibited a significant increased and/or association with tyrosine phosphorylation upon Src expression. These mainly include signaling, cytoskeleton and vesicular-associated proteins. Interestingly, Src also phosphorylated a cluster of tyrosine kinases, ie the receptors Met and EphA2, the cytoplasmic tyrosine kinase Fak and pseudo-tyrosine kinase $\operatorname{SgK} 223$, which were required for its invasive activity. Similar results were obtained with the metastatic Colo205 CRC cells that exhibit high endogenous Src activity. We concluded that Src uses a tyrosine kinases network to promote its invasive activity in $\mathrm{CRC}$ and this implicates a reverse signaling via tyrosine kinase receptors. Targeting these tyrosine kinases may be of significant therapeutic value in this cancer. 


\section{INTRODUCTION}

The non receptor tyrosine kinase Src plays important roles in cell proliferation, survival and migration induced by extracellular stimuli including growth factors and integrins (1). It also shows oncogenic activity when deregulated, a situation originally found with vSrc, the transforming product of the avian retrovirus RSV (2). Since then, Src has been reported deregulated in human cancer (3). Remarkably, elevated kinase activity has been found in more than $80 \%$ of $\mathrm{CRC}$ as compared to the normal counterpart and this has been associated with a poor clinical outcome (3). How Src is deregulated in this cancer has not been clearly established yet. An activating mutation has been reported in a minority of advanced tumors, suggesting the existence of alternative mechanisms for kinase deregulation (4). Accordingly, kinase activation often correlates with protein overexpression (2). This molecular event is however not sufficient in a normal cellular context as Src is subjected to strict regulation that keeps the enzyme in an inactive form. Therefore, it has been postulated that Src activation additionally implicates alteration of important Src regulators by genetic or epigenetic mechanisms, including the inhibitory C-terminal Src tyrosine kinase Csk (2).

A large body of evidence points to Src deregulation as an important event for colon tumorigenesis and metastasis (3). For instance, Src regulates growth, survival and invasion of some CRC cell-lines in vitro $(3,5)$. Moreover, it contributes to tumor growth, angiogenesis and metastasis in xenografts nude mouse models (3). Therefore, Src has become an attractive therapeutic target in this cancer and several small inhibitors are under clinical trials. Intriguingly, these Src oncogenic signaling have been unraveled only partially. While Src interacts with components of the focal contacts and the adherent junctions for cell migration (2), substrates important for cellular growth and invasiveness have been poorly described. MS-based quantitative phosphoproteomics has been a valuable tool to decipher signaling pathways initiated by a given tyrosine kinase (6). Among these, the Stable Isotope Labeling 
with Amino acids in Cell culture (SILAC) has been employed for the identification of oncogenic tyrosine kinase signaling such as HER2 (7) and Bcr-Abl (8). Here we used this approach to address Src oncogenic signaling in advanced CRC cells. By overexpressing Src in SW620 cells, we identified 136 substrates and/or tyrosine phosphorylation-associated proteins. These include proteins with signaling, cytoskeleton and vesicular trafficking functions, which may be important for the high Src invasiveness observed in these cells. Additionally, we identified a small cluster of tyrosine kinases that mediates this Src oncogenic signaling, uncovering a tyrosine kinases network important for the induction of cellular invasion. Targeting these tyrosine kinases may therefore be of significant value in advanced CRC therapy.

\section{MATERIAL AND METHODS}

Reagents. Human Src has been subcloned in pMX-pS-CESAR. Construct expressing Pragmin (a gift of Dr Negishi, Kioto University) and PragminY397F that was generated by mutagenesis have been subcloned into pBABE retroviral vectors. pRETRO-SUPER expressing shRNA used in this study were from TRANSAT (Saint-Priest, France). Targeting sequences inserted in shRNA constructs were GACACTCGGTAGTCTATAC (control), GAtGAgtTAtTAGCAGAAG (Syk), GAAGTCTAACTATGAAGTA (Fak), GCAGTATACGGAGCACTTC (EphA2), GTCACAGGCCAAGATAGAA (SgK223) and TAAGAGCTGTGAGAATATA (Met). siRNA targeting sequence were form Darmacon and incuded AATTCTCCGAACCTGTCACGT (control), AAAGATAAACCTCTCATAATG (met), AATGACATGCCGATCTACATG (EphA2) and AAGCTAGTGACGTATGGATGT

(Fak). ${ }^{13} \mathrm{C}_{6}{ }^{15} \mathrm{~N}_{4}$-Arg and ${ }^{13} \mathrm{C}_{6}{ }^{15} \mathrm{~N}_{2}$-Lys were from Invitrogen (Cergy, France) and Sigma Aldrich (St Quentin, France) respectively, anti-pTyr column from Upstate Inc (Charlottesville, USA), SU6656 and PP1 and PP2 from Calbiochem (La Jolla, CA), SU11274 
and BAY 61-3606 from Sigma Aldrich. Antibodies used in this study were 4G10 (42), pY100 (CST, Danvers, MA), ARAP3 (33), anti-Calponin (34), anti-p130Cas (Upstate Inc, Charlottesville, USA), anti- $\alpha-\delta$ Catenin (BD Biosciences), anti-Cbl (sc-170, Santa Cruz Biotech, CA) anti-CHC X22 (Alexis Biochemicals, San Diego, CA), anti-EphA2 (sc-924, Santa Cruz Biotech), anti-EphB2 (sc-28980, Santa Cruz Biotech), anti-ezrin (35), anti-Fak (Upstate Inc), anti-pY861Fak, (sc-16663, Santa Cruz Biotech), anti-Hrs A-5 (Alexis Biochemicals), anti-IRSp53 (36), anti-Met (sc-161, Santa Cruz Biotech), antipY1234/1235Met (Invitrogen, CA), anti-Moesin was generated against the purified protein as in (35), anti-Odin (37), anti-Radixin was generated against the purified protein as in (35), antiSam68 (38), anti-SgK223 (29) was raised against the GST-Pragmin N-terminus, anti-SgK269 3G6 (Abnova GmbH, Heidelberg, Germany), anti-SHIP1 (39), anti-SHP2 (sc-280, Santa Cruz Biotech), anti-Src family kinases (40), anti-p85a subunit of PI3K (41), anti-PLC $\gamma$ (42), antipY418Src (Biosource), anti-Stam1/2 (43), anti-Syk (sc-1240, Santa Cruz Biotech), antipY325Syk and anti-pY524/525Syk (CST, Danvers, MA), anti-tubulin (42), anti-Vav2 (sc20803, Santa Cruz Biotech), anti-Wave2 (36), anti-ZO1 (sc-10804, Santa Cruz Biotech), antiZO2 (sc-11448, Santa Cruz Biotech).

Cell infections, growth, migration and invasion. SW620 and Colo205 cells (ATCC, Rockville, MD) were grown, infected and selected as described in (44). This procedure allows the generation of stable expressors in a polyclonal background. Src expressors were isolated by FACS and shRNA expressing cells by puromycin $(1 \mu \mathrm{g} / \mathrm{ml})$ selection. Standard cell growth was performed in $2 \%$ serum medium and measured by Sulforhodamide B staining (Sigma Aldrich). Soft-agar cell growth was performed as in (44). After 18-21 days colonies having $>50$ cells were scored as positive. Cells were treated every day for standard conditions and every week for soft-agar assays, with vehicle or indicated drug. Cell migration and 
invasion assays were performed in Boyden chambers (BD Bioscience, Le Pont de Claix, France) using 50,000 and 100,000 cells respectively, and in the presence of $100 \mu 1$ of Matrigel (2 $\mathrm{mg} / \mathrm{ml})$ (BD Bioscience) for invasion assays.

Growth of SW620 xenografts in nude mice. In vivo experiments were performed in compliance with the French guidelines for experimental animal studies (Direction des Services Vétérinaires, Ministère de l'Agriculture, agreement no. B 34-172-27) and fulfil the U.K. Coordinating Committee on Cancer Research guidelines for the welfare of animals in experimental neoplasia. Swiss nu/nu (nude) mice (Charles River, L'Arbresle, France) were injected s.c. with $2.10^{6}$ of SW620 cells infected with the control virus in one flank and with the same amount of cells infected with the Src virus on the opposite side. Tumor growth was assessed by tumor volume and tumor mass measurement at indicated days.

Mass Spectrometry analysis. SILAC $\left({ }^{13} \mathrm{C}_{6}{ }^{15} \mathrm{~N}_{4}\right.$-Arg- and ${ }^{13} \mathrm{C}_{6}{ }^{15} \mathrm{~N}_{2}$-Lys as heavy labeling), phosphotyrosine immunoaffinity purification (using a mixture of a mixture of $4 \mathrm{G} 10$ and pY100 antiboies), and tryptic digests were essentially performed as described in (11) except that cells $\left(2.10^{8}\right)$ were treated with the tyrosine phosphatases inhibitor orthovanadate $(1 \mathrm{mM})$ for $1 \mathrm{~h}$ before lysis to increase tyrosine phosporylation content. $50 \mathrm{mM} \beta$-octylglucoside was also added in the $1 \%$ Triton $\mathrm{X}-100$ lysis buffer to increase protein solubilization. Purified proteins were next separated on a SDS-PAGE gel. Trypsin-digested samples $(1 \mu \mathrm{l})$ obtained from 44 cut gel slices were next analyzed online using nanoflow HPLC-nano-electrospray ionization on a quadrupole time-of-flight (Q-TOF) mass spectrometer (QSTAR Pulsar-i, Applied Biosystems, Foster City, CA) coupled with an Ultimate 3000 HPLC (Dionex, Amsterdam, Netherland). Desalting and pre-concentration of samples were effectued on-line on a Pepmap ${ }^{\circledR}$ precolumn $(0.3 \mathrm{~mm}$ x $10 \mathrm{~mm})$. A gradient consisting of $0-40 \% \mathrm{~B}$ in $60 \mathrm{mn}$, 
$80 \% \mathrm{~B}$ in $15 \min (\mathrm{A}=0.1 \%$ formic acid, $2 \%$ acetonitrile in water $; \mathrm{B}=0.1 \%$ formic acid in acetonitrile) at $300 \mathrm{nl} / \mathrm{min}$ was used to elute peptides from the capillary $(0.075 \mathrm{~mm} \mathrm{x} 150 \mathrm{~mm})$ reverse-phase column $\left(\right.$ Pepmap $^{\circledR}$, Dionex), fitted with an uncoated silica PicoTip Emitter (NewOjective, Woburn, USA) with an outlet diameter of $8 \mu \mathrm{m}$. Spectra were recorded using the Analyst QS 1.1 software (Applied Biosystems). Parameters were adjusted as follows: ion spray voltage (IS), $1800 \mathrm{~V}$; curtain gas (CUR), 25; declustering potential (DP), $75 \mathrm{~V}$; focusing potential (FP), $265 \mathrm{~V}$; declustering potential 2 (DP2), $15 \mathrm{~V}$. Spectra were acquired with the instrument operating in the information dependant acquisition mode throughout the HPLC gradient. Every $7 \mathrm{~s}$, the instrument cycled through acquisition of a full-scan spectrum (1 s) and two MS/MS spectra (3 s each). Peptides fragmentation was performed using nitrogen gas on the most abundant doubly or triply charged ions detected in the initial MS scan, with a collision energy profile optimized according to peptide mass (using manufacturer parameters), and an active exclusion time of $0.60 \mathrm{~min}$. All MS/MS spectra were searched against the Homo

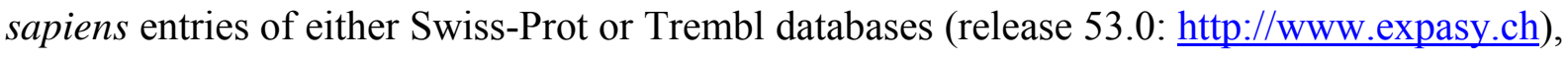
by using the Mascot v 2.1 algorithm (http://www.matrixscience.com). Search parameters were mass accuracy $0.1 \mathrm{Da}$ for MS and MS/MS data ; 1 miscleavage ; variable modifications: oxidized methionine, Phospho (Y), SILAC-labels : Lys-8 and Arg-10. All significant hits $(\mathrm{p}<0.05)$ were manually inspected. Quantification was performed by using MSQuant v1.4.1 software developed by Mann and colleagues (http://msquant.sourceforge.net). Data were manually inspected and corrected when necessary. All significant hits were manually inspected.

Biochemistry. Immunoprecipitation, Western blotting and siRNA transfections were performed as described in (44). Imunoprecipitates (ip, when indicated) or 20 to $50 \mu \mathrm{g}$ of proteins of a whole cell lysate were loaded per lane on 9\% SDS-PAGE gels and then 
transferred onto Immobilon membranes (Milllipore) as previously described. Detection was performed using the ECL system (Amersham). Optimal exposure times of membranes were used and protein expression and phosphorylation was quantified using ImageQuant TL software (Molecular Dynamics), and adjusted for background noise and protein loading.

\section{RESULTS}

\section{Src promotes growth and invasiveness of the SW620 CRC cells.}

We first transduced wild-type Src retrovirally in the metastatic CRC SW620 cells that exhibit a moderate level of endogenous Src as compared to the metastatic Colo205 cells (9). A >5 fold increase in Src protein level was obtained (Fig. 1A), which induced a strong increase in tyrosine phosphorylation content (Fig. 2A) together with significant cellular oncogenic activity: increased cell growth was observed both in standard (Fig. 1B) and soft agar conditions (Fig. 1C). Similarly, Src increased tumor growth by 3 fold in mouse xenograft assays (Supplementary Fig. S1). While Src moderately increased cell motility (Fig. 1D, left panel), it induced remarkable cell invasiveness (Fig. 1D, right panel). This cellular activity was still dependent upon Src activity as it was inhibited by the Src-like inhibitor SU6656 (10) (Fig. 1D, right panel). This data supports the hypothesis that the induction of the Src level is sufficient to induce its oncogenic activity even at late stage of tumorigenesis

\section{Quantitative Src phosphoproteomics in CRC cells.}

We next sought Src substrates important for oncogenic signaling by quantitative phosphoproteomics using the SILAC approach. SW620-Src cells were labeled with "heavy" ${ }^{13} \mathrm{C}_{6}{ }^{15} \mathrm{~N}_{4}$-Arg- and ${ }^{13} \mathrm{C}_{6}{ }^{15} \mathrm{~N}_{2}$-Lys-containing medium while SW620 cells were cultured in "light" normal conditions. Cell lysates were next combined, and phosphotyrosine-containing proteins were purified using anti-phosphotyrosine antibodies. Proteins were separated by 
SDS/PAGE, digested with trypsin, and subjected to liquid chromatography-tandem MS (11). Quantification was made from the relative intensities of Arg/Lys labeled tryptic peptides. With this approach, we obtained 467 proteins, 136 of which exhibiting increased, and 13 reduced, tyrosine phosphorylation, upon Src expression (Fig. 2B and Supplementary Table S1). The small number of reduced phosphorylations was attributed to the cellular treatment of the tyrosine phosphatases inhibitor orthovanadate performed shortly before lysis in order to increase tyrosine phosphorylation content. Src-induced phosphorylation was next confirmed biochemically for $32 / 35$ of them (Fig. 3 and 4A), suggesting that more than $90 \%$ of the identified candidates are indeed Src substrates and thus validates the SILAC analysis. The absence of increased tyrosine phosphorylation content for 3 of them may be due to an increased association with Src substrates or/and the inability of the used antibodies to recognize the phosphorylated form of the protein. 41 of identified proteins have signaling, and 31 cytoskeletal functions (supplementary Table S2). Of some note are the CDCP1/PKC $\delta$ transmembrane signaling complex involved in survival (12), the E-cadherin/catenins complexes in mesenchyme induction and cell motility (2), and proteins of the ERM family in cell invasion (13). Additionally, Src targets the Wave2 and the alpha6/beta4 integrin complexes, recently identified as novel players of epithelial cell motility/invasion $(14,15)$. We also noticed a subgroup of signaling proteins that regulate the small GTPases of the Rho family, and which may modulate actinic cytoskeleton occurring during cell invasion (13). These include the Rho GAP ARAP1, 3 and p200 Rho GAP, and the Rho GEF Vav2 and FGD5, which has not been previously identified as a Src substrate. Unexpectedly, we found a third group of 23 Src targets with vesicular trafficking functions (supplementary Table S2). This was exemplified with components of the COPII complex of the secretory pathway (16) and VHS-containing proteins (Hrs and Tom1L1), involved in transmembrane proteins trafficking/signaling (17). 


\section{Src phosphorylates a cluster of tyrosine kinases important for cell invasiveness.}

The SILAC analysis confirmed a 6 fold increase in Src expression showing accuracy of the method, but it additionally identified a cluster of 7 protein tyrosine kinases and pseudotyrosine kinases as potential Src substrates (Table 1). This included the receptors Met, EphA2 and EphB2, and the cytoplasmic tyrosine kinases Fak and Syk and pseudokinases SgK223 and SgK269. Increased tyrosine phosphorylation was confirmed for 5 of them biochemically, ie Met, EphA2, Fak, Syk and SgK223 (Fig. 4A), and this correlated with a Src complex formation (not shown). Similar results were obtained from cells that were not treated with the phosphatase inhibitor orthovanadate, indicating that these phosphorylations were specific to Src expression (Supplementary Fig. S2A). We next investigated their role in Src-induced tyrosine phosphorylation. To this end, respective tyrosine kinase was inactivated by stably expressing a shRNA leading to $>50 \%$ reduction of both mRNA (not shown) and protein levels (Fig. 4B) in SW620-Src cells. We observed that tyrosine phosphorylation was significantly reduced in cells with inactive SgK223 and Syk, and at a lower level in cells with inactive Met. Accordingly, Src induced Met and Syk activation as shown by the increase level in the phosphorylated and activated form of these kinases (Supplementary Fig. S2B). Therefore Src

may use these TyrK activities to induce maximal phosphorylation in these CRC cells. Surprisingly, Src specific activity was also reduced in these cells with inactive Met, EphA2, Syk and SgK223, suggesting the existence of interplay between Src and these TyrK for signaling (Fig. 4C). The function for these kinases was next evaluated on Src oncogenic activity. We found that Src-induced anchorage-independent cell growth was strongly inhibited in all cell-lines, except for Met (Fig. 4D). However, none of them had a significant role in Src-induced cell proliferation (Supplementary Fig. S3), indicating that these TyrK may 
impact on survival signaling rather than cell-cycle progression, the later being probably ensured by phosphorylation of direct Src substrates.

The role for these TyrK was next evaluated on Src-induced invasiveness. We found that this cellular activity was strongly inhibited in all cell-lines with down-regulated tyrosine kinases, except Syk (Fig. 5A, left panel). Similar results were obtained from SW620-Src cells that were transiently transfected with specific siRNA targeting Met, EphA2 and Fak, indicating that these inhibitory effects were not due to off-targets and/or long-term depletion of these kinases (Supplementary Fig. S4C and S4D). In contrast, they had a low impact on the residual invasiveness of SW620 cells, confirming that their role is tightly related to Src activity (Fig S4A and B). The absence of Syk function was next confirmed with the orally available Syk catalytic inhibitor BAY 61-3606 (18), that did not affect invasiveness (Fig. 5A, right panel), while reducing Syk tyrosine phosphorylation (Fig. 6C, bottom panel). In contrast, the Met inhibitor SU11274 (19) (Fig. 6C, upper panel) blocked this cellular response (Fig. $5 \mathrm{~A}$, right panel), confirming the requirement of Met in this Src oncogenic function. It should be mentioned that BAY 61-3606 strongly inhibited cellular proliferation, in contrast to what observed in cells with reduced Syk (Supplementary Fig. S3). This compound may therefore target an additional kinase important for cellular proliferation to be identified.

Since very few information is available on $\mathrm{SgK} 223$, we wished to address its invasive function in more details. To this end, the rat ortholog Pragmin was reintroduced in SW620Src cells with reduced Sgk223, which was not targeted with the used shRNA sequence. Pragmin largely restored Src invasive activity, confirming a specific function for SgK223 in this signaling process (Fig. 5B and 5C). Whether this uncovered function was regulated by Src was next investigated. The phosphosite data base http://www.phosphosite.org revealed that SgK223 was frequently phosphorylated on Tyr413. Accordingly, we found that Src induced a robust phosphorylation of Pragmin in these cells, unlike PragminY391F, in which 
the corresponding Tyr413 present in Pragmin was replaced by Phe (Fig. 5B). At the functional level, PragminY391F had a reduced rescuing effect in these cells (Fig. 5C). This set of data strongly suggested Src invasive signaling implicates phosphorylation of SgK223 on Tyr413 in advanced CRC cells.

Finally, we wished to confirm these data in cells expressing high endogenous Src, with the example of the metastatic CRC cell-line Colo205. As observed with SW620-Src, these cells required a Src-like activity to induce cellular invasion (Fig. 6D). We found that the tyrosine kinases Met, EphA2, Fak, Syk were also phosphorylated in a Src-like dependent manner, as suggested with the Src-like inhibitors SU6656, PP1 and PP2 (Fig. 6A). The variable effect of SU6656 and PP1 on these biochemical events were attributed to their capacity to target distinct pools of Src present in these cells rather than the targeting of additional kinases (not shown). SgK223 protein phosphorylation could not be assessed in these cells. This may probably due to the moderate efficacy of our antibodies and the low level of the expressed protein as observed on its mRNA level (not shown). At the functional level, all these phosphorylated TyrK, but not Syk, were required for cell invasion: downregulation of respective kinases (Fig. 6B) with the same shRNA strategy gave strong inhibitory effects (Fig. 6D). Thus, Src uses a similar TyrK network to induce its invasive activity in Colo205 cells, suggesting the existence of a common TyrK signaling network for oncogenic induction in advanced CRC cells.

\section{DISCUSSION}

Here we provide new insights into Src oncogenic activity in CRC. Firstly, our data show that normal Src overexpression can induce high invasiveness of advanced cancer cells in vitro, in close correlation with the proposed role for Src in CRC metastasis (3). Secondly, applied quantitative SILAC phosphoprotemics uncovered several features of Src signaling in 
these cells, including phosphorylation/interaction of components of phosphoinositides and Rho GTPases-dependent pathways and regulators of F-actin, microtubule and vesicular trafficking networks. These data also show that Src targets more than a dozen of plasma membrane proteins, several of them having ligand, receptor/co-receptor functions related to cellular growth, survival and adhesion. Therefore, Src may use a wide range of reverse signaling processes important for its oncogenic activity as recently suggested with the Met receptor (5).

Another important insight from this study relies on the interaction of Src with a cluster of tyrosine kinases required for cell invasion. Src may thus require additional tyrosine kinases to phosphorylate components of the invasive pathway. This cluster comprises the receptors Met and EphA2, previously reported interacting with Src $(20,21)$ and being implicated in CRC tumorigenesis $(22,23)$. Moreover, our functional studies demonstrate that they mediate Src oncogenic activity in agreement with a reverse signaling process operating in these cells. It also includes the cytoplasmic tyrosine kinase Fak, which has been largely implicated in Srcinduced cell motility and invasion (24). Interestingly, Fak does not impact on the global tyrosine phosphorylation content in contrast to the other members of the cluster, suggesting that either it phosphorylates a discrete group of substrates or it has an adaptor function, as previously reported (24). More surprisingly is the identification of Syk as a Src substrate in these cancer cells, which is inconsistent with its tumor suppressor role described in breast cancer (25). Therefore, Syk may have distinct functions dependent on the tumor origin. In supporting this idea, Syk regulates Src-induced tyrosine phosphorylation and anchorageindependent growth of SW620-Src cells. This is further supported with the function recently reported in squamous cell carcinoma of the head and neck (26). Finally, we show that Src also uses $\operatorname{SgK} 223$ for its invasive activity. $\operatorname{SgK} 223$ belongs to the $10 \%$ of catalytic-inactive kinases present in the human kinome (27) that nevertheless can have important oncogenic 
function. This has been first highlighted with the EGF receptor member HER3 in breast cancer. HER3 exhibits a co-receptor function that boosts signaling induced by other receptors of the family (28). Very few information is however available for SgK223. It belongs to the NFK3 sub-family that also includes SgK269, with unknown functions (27). Interestingly, SgK223 has been recently identified as an important effector of the small GTPase Rnd2 for Rho-dependent F-actin assembly (29), which would fit with its pro-invasive activity in CRC cells. While it does not have apparent intrinsic tyrosine kinase activity (not shown), SgK223 strongly impacts on Src-induced tyrosine phosphorylation in cellulo. We thus hypothesize that it regulates Src phosphorylation events important for Rho-dependent signaling. Overall, these results assign an unanticipated role for $\mathrm{SgK} 223$ in Src oncogenic signaling and suggest an involvement of this pseudo-kinase in CRC advanced tumorigenesis.

Finally, our report suggests that Src orchestrates a tyrosine kinases signaling network for invasiveness. This notion has been recently suggested with the example of two recent phosphoproteomic studies revealing the phosphorylation of cluster of the TyrK FAK, EphA2, Met and ACK in Src-transformed mouse fibroblasts (30) and the cluster of TyrK including Met and EphA2 in non small cell lung cancer lines expressing activated ROS, ALK, PDGFR $\alpha$ and EGFR(31). Nevertheless, the function for these TyrK networks has not been investigated. These reports together with our results thus suggest the involvement of a common TyrK signaling network operating during neoplastic transformation initiated by a deregulated TyrK. Interestingly, our data additionally indicate that Met, Syk and SgK223 may in turn further activate Src, uncovering an interplay between Src and these tyrosine kinases. How Src interacts with these tyrosine kinases is an important issue that can be addressed by phosphoproteomics. Similarly, these results raise the interesting idea that Src deregulation could originate, at least in part, from the complex array of phosphorylated/activated tyrosine 
kinase receptors recently observed in other solid tumors with important oncogenic activities (32); thus targeting Src activity could be of therapeutic value in these cancers. 
Disclosure of Potential Conflicts of Interest

No potential conflicts of interest were disclosed. 


\section{ACKNOWLEDGEMENTS}

We thank P. Coopman, P. Mangeat, F. Hollande, C. Gauthier-Rouvière, B. Payrastre, G. Scita, E. Stradal, P. Lock and M. Negishi for various reagents, S. Perrier and M. Brissac for technical assistance and members of the laboratory for helpful discussion. This work was supported by the CNRS, University of Montpellier 1 and 2, Association pour la Recherche contre le Cancer (ARC, $\mathrm{n}^{\circ}$ 4025), Institut National du Cancer INCa and the Canceropole GSO. CL is supported by the Ligue Contre de Cancer, LCC, and AS by INCa. SR is an INSERM investigator. 


\section{REFERENCES}

1. Thomas SM, Brugge JS. Cellular functions regulated by Src family kinases. Annu Rev Cell Dev Biol 1997;13:513-609

2. Yeatman TJ. A renaissance for SRC. Nat Rev Cancer 2004;4: 470-80.

3. Summy JM, Gallick GE. Src family kinases in tumor progression and metastasis. Cancer Metastasis Rev 2003;22:337-58.

4. Irby RB, Mao W, Coppola D, Kang J, Loubeau JM, Trudeau W, et al. Activating SRC mutation in a subset of advanced human colon cancers. Nat Genet 1999;21:187-90.

5. Emaduddin M, Bicknell DC, Bodmer WF, Feller SM. Cell growth, global phosphotyrosine elevation, and c-Met phosphorylation through Src family kinases in colorectal cancer cells. Proc Natl Acad Sci U S A 2008;105:2358-62.

6. Schmelzle K, White, FM. Phosphoproteomic approaches to elucidate cellular signaling networks. Curr Opin Biotechnol 2006;17: 406-14.

7. Bose R, Molina H, Patterson AS, Bitok JK, Periaswamy B, Bader JS, et al. Phosphoproteomic analysis of Her2/neu signaling and inhibition. Proc Natl Acad Sci U S A 2006;103:9773-8.

8. Liang X, Hajivandi M, Veach D, Wisniewski D, Clarkson B, Resh MD, et al. Quantification of change in phosphorylation of BCR-ABL kinase and its substrates in response to Imatinib treatment in human chronic myelogenous leukemia cells. Proteomics 2006;6:4554-64.

9. Dehm S, Senger MA, Bonham K. SRC transcriptional activation in a subset of human colon cancer cell lines. FEBS Lett 2001;487:367-71. 
10. Laird AD, Li G, Moss KG, Blake RA, Broome MA, Cherrington JM, et al. Src family kinase activity is required for signal tranducer and activator of transcription 3 and focal adhesion kinase phosphorylation and vascular endothelial growth factor signaling in vivo and for anchorage-dependent and -independent growth of human tumor cells. Mol Cancer Ther 2003;2:461-9.

11. Amanchy R, Kalume DE, Iwahori A, Zhong J, Pandey, A. Phosphoproteome analysis of HeLa cells using stable isotope labeling with amino acids in cell culture (SILAC). $\mathrm{J}$ Proteome Res 2005;4:1661-71.

12. Uekita T, Jia L, Narisawa-Saito M, Yokota J, Kiyono T, Sakai R. CUB domaincontaining protein 1 is a novel regulator of anoikis resistance in lung adenocarcinoma. Mol Cell Biol 2007;27:7649-60.

13. Sahai E, Marshall, CJ. Differing modes of tumour cell invasion have distinct requirements for Rho/ROCK signalling and extracellular proteolysis. Nat Cell Biol $2003 ; 5: 711-9$.

14. Iwaya K, Oikawa K, Semba S, Tsuchiya B, Mukai Y, Otsubo T, et al. Correlation between liver metastasis of the colocalization of actin-related protein 2 and 3 complex and WAVE2 in colorectal carcinoma. Cancer Sci 2007;98:992-9.

15. Gimona M, Buccione R, Courtneidge SA, Linder S. Assembly and biological role of podosomes and invadopodia. Curr Opin Cell Biol 2008;20:235-41.

16. Sato K, Nakano A. Mechanisms of COPII vesicle formation and protein sorting. FEBS Lett 2007;581:2076-82.

17. Lohi O, Poussu A, Mao Y, Quiocho F, Lehto VP. VHS domain -- a longshoreman of vesicle lines. FEBS Lett 2002;513:19-23. 
18. Yamamoto N, Takeshita K, Shichijo M, Kokubo T, Sato M, Nakashima K, et al. The orally available spleen tyrosine kinase inhibitor 2-[7-(3,4-dimethoxyphenyl)imidazo[1,2-c]pyrimidin-5-ylamino]nicotinamide dihydrochloride (BAY 61-3606) blocks antigen-induced airway inflammation in rodents. J Pharmacol Exp Ther 2003;306:1174-81.

19. Sattler M, Pride YB, Ma P, Gramlich JL, Chu SC, Quinnan LA, et al. A novel small molecule met inhibitor induces apoptosis in cells transformed by the oncogenic TPRMET tyrosine kinase. Cancer Res 2003;63:5462-9.

20. Ponzetto C, Bardelli A, Zhen Z, Maina F, dalla Zonca P, Giordano S, et al. A multifunctional docking site mediates signaling and transformation by the hepatocyte growth factor/scatter factor receptor family. Cell 1994;77:261-71.

21. Fang WB, Ireton RC, Zhuang G, Takahashi T, Reynolds A, Chen J. Overexpression of EPHA2 receptor destabilizes adherens junctions via a RhoA-dependent mechanism. $\mathrm{J}$ Cell Sci 2008;121:358-68.

22. Birchmeier C, Birchmeier W, Gherardi E, Vande Woude GF. Met, metastasis, motility and more. Nat Rev Mol Cell Biol 2003;4:915-25.

23. Merlos-Suarez A, Batlle E. Eph-ephrin signalling in adult tissues and cancer. Curr Opin Cell Biol 2008;20:194-200.

24. Mitra SK, Schlaepfer D. Integrin-regulated FAK-Src signaling in normal and cancer cells. Curr Opin Cell Biol 2006;18:516-23.

25. Coopman PJ, Do MT, Barth M, Bowden ET, Hayes AJ, Basyuk E, et al. The Syk tyrosine kinase suppresses malignant growth of human breast cancer cells. Nature 2000;406:742-7. 
26. Luangdilok S, Box C, Patterson L, Court W, Harrington K, Pitkin L, et al. Syk tyrosine kinase is linked to cell motility and progression in squamous cell carcinomas of the head and neck. Cancer Res 2007;67:7907-16.

27. Manning G, Whyte, DB, Martinez R, Hunter T, Sudarsanam S. The protein kinase complement of the human genome. Science 2002;298:1912-34.

28. Sithanandam G, Anderson LM. The ERBB3 receptor in cancer and cancer gene therapy. Cancer Gene Ther 2008;15:413-48.

29. Tanaka H, Katoh H, Negishi M. Pragmin, a novel effector of Rnd2 GTPase, stimulates RhoA activity. J Biol Chem 2006;281:10355-64.

30. Luo W, Slebos RJ, Hill S, Li M, Brabek J, Amanchy R, et al. Global impact of oncogenic Src on a phosphotyrosine proteome. J Proteome Res 2008;7:3447-60.

31. Rikova K, Guo A, Zeng Q, Possemato A, Yu J, Haack H, et al. Global survey of phosphotyrosine signaling identifies oncogenic kinases in lung cancer. Cell 2007; 131:1190-203.

32. Stommel JM, Kimmelman AC, Ying H, Nabioullin R, Ponugoti AH, Wiedemeyer R, et al. Coactivation of receptor tyrosine kinases affects the response of tumor cells to targeted therapies. Science 2007;318:287-90.

33. I ST, Nie Z, Stewart A, Najdovska M, Hall NE, He H, et al. ARAP3 is transiently tyrosine phosphorylated in cells attaching to fibronectin and inhibits cell spreading in a RhoGAP-dependent manner. J Cell Sci 2004;117:6071-84.

34. Represa A, Trabelsi-Terzidis H, Plantier M, Fattoum A, Jorquera I, Agassandian C, et al. Distribution of caldesmon and of the acidic isoform of calponin in cultured cerebellar neurons and in different regions of the rat brain: an immunofluorescence and confocal microscopy study. Exp Cell Res 1995;221:333-43. 
35. Andreoli C, Martin M, Le Borgne R, Reggio H, Mangeat P. Ezrin has properties to self-associate at the plasma membrane. J Cell Sci 1994;107:2509-21.

36. Disanza A, Mantoani S, Hertzog M, Gerboth S, Frittoli E, Steffen A, et al. Regulation of cell shape by Cdc42 is mediated by the synergic actin-bundling activity of the Eps8IRSp53 complex. Nat Cell Biol 2006;8:1137-47.

37. Pandey A, Blagoev B, Kratchmarova I, Fernandez M, Nielsen M, Kristiansen TZ, et al. Cloning of a novel phosphotyrosine binding domain containing molecule, Odin, involved in signaling by receptor tyrosine kinases. Oncogene 2002;21:8029-36.

38. Lock P, Fumagalli S, Polakis P, McCormick F, Courtneidge SA. The human p62 cDNA encodes Sam68 and not the RasGAP-associated p62 protein. Cell 1996;84:234.

39. Giuriato S, Bodin S, Erneux C, Woscholski R, Plantavid M, Chap H, et al. pp60c-src associates with the SH2-containing inositol-5-phosphatase SHIP1 and is involved in its tyrosine phosphorylation downstream of alphaIIbbeta3 integrin in human platelets. Biochem J 2000;348:107-12.

40. Roche S, Koegl M, Barone MV, Roussel MF, Courtneidge SA. DNA synthesis induced by some but not all growth factors requires Src family protein tyrosine kinases. Mol Cell Biol 1995;15:1102-9.

41. Roche S, Koegl M, Courtneidge SA. The phosphatidylinositol 3-kinase alpha is required for DNA synthesis induced by some, but not all, growth factors. Proc Natl Acad Sci U S A 1994;91:9185-9.

42. Roche S, McGlade J, Jones M, Gish GD, Pawson T, Courtneidge SA. Requirement of phospholipase C gamma, the tyrosine phosphatase Syp and the adaptor proteins Shc and Nck for PDGF-induced DNA synthesis: evidence for the existence of Rasdependent and Ras-independent pathways. Embo J 1996;15:4940-8. 
43. Franco M, Furstoss O, Simon V, Benistant C, Hong WJ, Roche S. The adaptor protien Tom1L1 is a negative regulator of Src mitogenic signaling induced by growth factors. Mol Biol Cell 2006;26:1932-47.

44. Sirvent A, Boureux A, Simon V, Leroy C, Roche S. The tyrosine kinase Abl is required for Src-transforming activity in mouse fibroblasts and human breast cancer cells. Oncogene 2007;26:7313-23. 
Table1. List of Tyrosine kinases targeted by Src.

\begin{tabular}{lc} 
Tyrosine kinase & $\begin{array}{c}\text { fold change with Src } \\
\text { expression }\end{array}$ \\
\hline Src & 6.27 \\
Syk & 4.44 \\
EphA2 & 2.52 \\
SgK223 & 2.34 \\
Met & 2.12 \\
EphB2 & 1.74 \\
SgK269 & 1.62 \\
\hline
\end{tabular}




\section{FIGURE LEGENDS}

\section{Figure 1. Src promotes cell growth and invasiveness of SW620 cells.}

A, Western blot analysis showing the Src level in cells infected with control (mock) and Src expressing retroviruses as indicated. The level of tubulin is also shown. $B, C$, Src expression promotes growth of SW620 cells in standard medium and soft-agar condition. $B$, time-course of cell growth (arbitrary units) in standard conditions. C, left, a representative example of colonies obtained with cells infected with control (mock) and Src expressing retroviruses in soft-agar medium at day 20. C, right, statistical analysis of colonies number of cells infected with indicated retrovirus and treated with $5 \mu \mathrm{M}$ SU6656 as shown. $D$, Src promotes cellular migration (left panel) and invasion (right panel) through matrigel of SW620 cells in vitro. Number of cells/field that have migrated $(C)$ or invaded through matrigel $(D)$ in Boyden chamber assays. Cells have been treated with $5 \mu \mathrm{M}$ SU6656 when indicated. The mean \pm SD $(\mathrm{n}>3)$ is shown.

\section{Figure 2. Src phosphoproteomic analysis in SW620 cells using SILAC.}

A, Western blot showing the tyrosine phosphorylation content of cells infected with the shown retroviruses and treated or not with orthovanadate as indicated. Western blotting from indicated p-Tyr immunoprecipitations. $B$, quantification of phosphorylations by SILAC. Summary of fold change with Src for 467 proteins shown in Supplementary Table S1, with several individual proteins indicated. Proteins with ratio $>1.3$ (upper line) are considered as increased and $<0.7$ (lower line) as decreased in their tyrosine phosphorylation content.

\section{Figure 3. Validation of Src-induced protein tyrosine phosphorylation by immunoprecipitation and Western blotting.}


Level and tyrosine phosphorylation content of selected Src targets as identified by phosphoproteomics. Western blotting analysis of tyrosine phosphorylation content and level of indicated protein that was immunoprecipitated from vanadate treated cells infected with shown virus and using indicated antibodies. $A$, signaling proteins; $B$, cytoskeletal associated proteins; $C$, vesicular trafficking associated proteins.

Figure 4. Src targets a cluster of tyrosine kinases important for increased tyrosine phosphorylation content and the promotion of anchorage-independent cell growth.

A, Tyrosine kinases with increased tyrosine phosphorylation content upon Src expression. Western blot showing the tyrosine phosphorylation and the protein levels in indicated tyrosine kinase immunoprecipitates from cells overexpressing Src or not, as shown. B, Western blot showing the level of indicated tyrosine kinases obtained from lysates of SW620-Src cells infected with retrovirus expressing indicated shRNA. The level of tubulin is also shown. $C$, Western blot showing the tyrosine phosphorylation content of cell overexpressing or not Src as shown and infected with indicated shRNA. D, left, and example, right and statistical analysis of anchorage-independent cell growth (number of colonies) of SW620-Src cells infected with retroviruses expressing indicated shRNA. The mean $\pm \operatorname{SD}(n>3)$ is shown

Figure 5. Src targets a cluster of tyrosine kinases important he promotion of cellular invasiveness

A, left, invasiveness of SW620-Src cells infected with retroviruses expressing indicated shRNA. Right, invasiveness of SW620-Src cells treated with vehicle (DMSO), $5 \mu$ M SU6656, $2 \mu \mathrm{M}$ SU11274 or $5 \mu \mathrm{M}$ BAY 61-3606 as indicated. Number of cells/field that have invaded through matrigel in Boyden chamber assays. $B$, Src phosphorlyates the rat ortholog of 
SgK223, Pragmin, at Tyr391 in SW620-Src cells. Tyrosine phosphorylation and protein content of immunoprecipitated Pragmin mutants that were retrovirally transduced in SW620Src cells expressing SgK223 specific shRNA as indicated. C, Pragmin but not PragminY391F restores invasiveness in SW620-Src cells with reduced SgK223. Number of cells/field that have invaded through matrigel in Boyden chamber assays. The mean $\pm \operatorname{SD}(n>3)$ is shown.

\section{Figure 6. Src targets a similar cluster of tyrosine kinases for the promotion of invasiveness of Colo205 cells.}

A, Src phosphorylates a similar cluster of tyrosine kinases in Colo205 cells. Western blot of the tyrosine phosphorylation content of shown immunoprecipitated tyrosine kinases from cells treated with indicated drugs $(5 \mu \mathrm{M})$. B, Western blot showing the level of indicated tyrosine kinase level obtained from lysates of Colo205 cells infected with retrovirus expressing indicated shRNA. The level of tubulin is also shown. $C$, inhibition of Met and Syk phosphorylation by SU11274 and BAY 61-3606 respectively. Western blot showing the tyrosine phosphorylation and protein level of indicated tyrosine kinases that were imunoprecipitated from Colo205 cells treated as indicated for 3 h. $D$, invasiveness of Colo205 cells infected with retroviruses expressing indicated shRNA or treated with indicated drugs (5 $\mu \mathrm{M}$ SU6656, $2 \mu \mathrm{M}$ SU11274 or $5 \mu \mathrm{M}$ BAY 61-3606). Number of cells/field that have invaded through matrigel in Boyden chamber assays. The mean $\pm \mathrm{SD}(\mathrm{n}>3)$ is shown. 


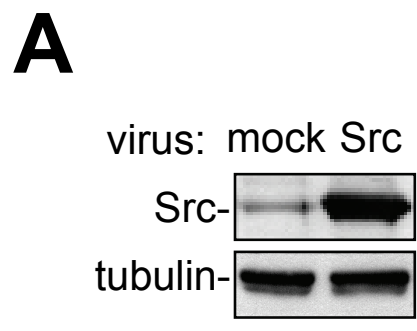

C
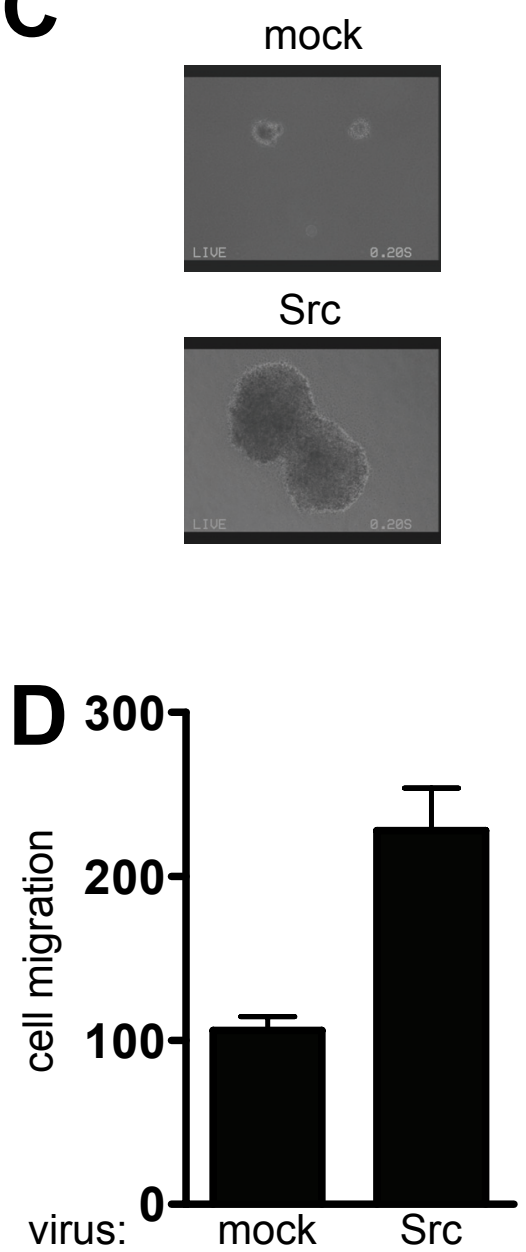
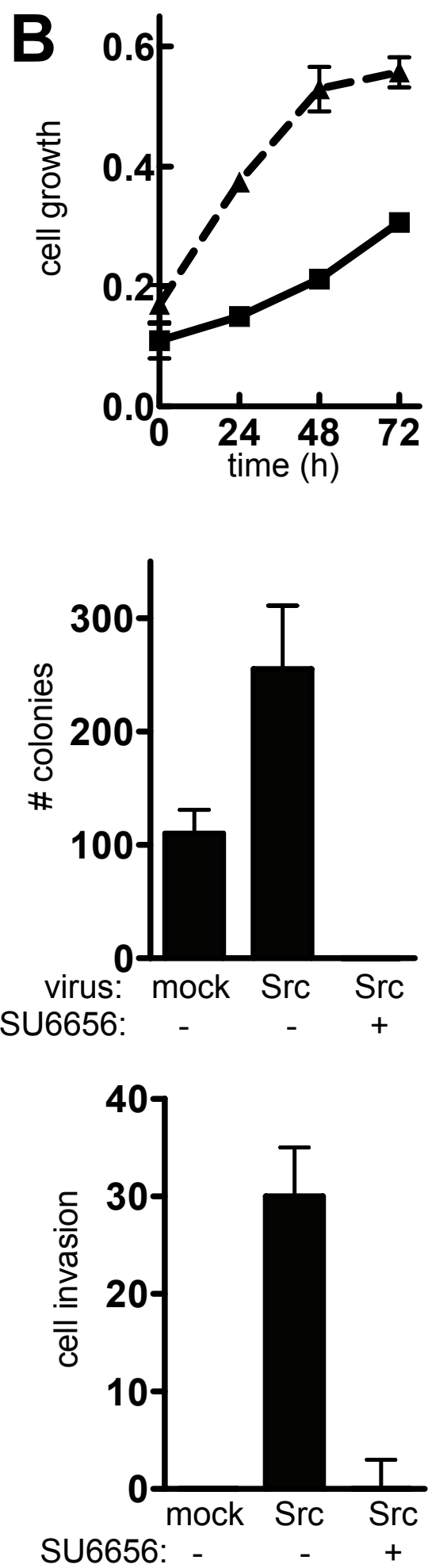

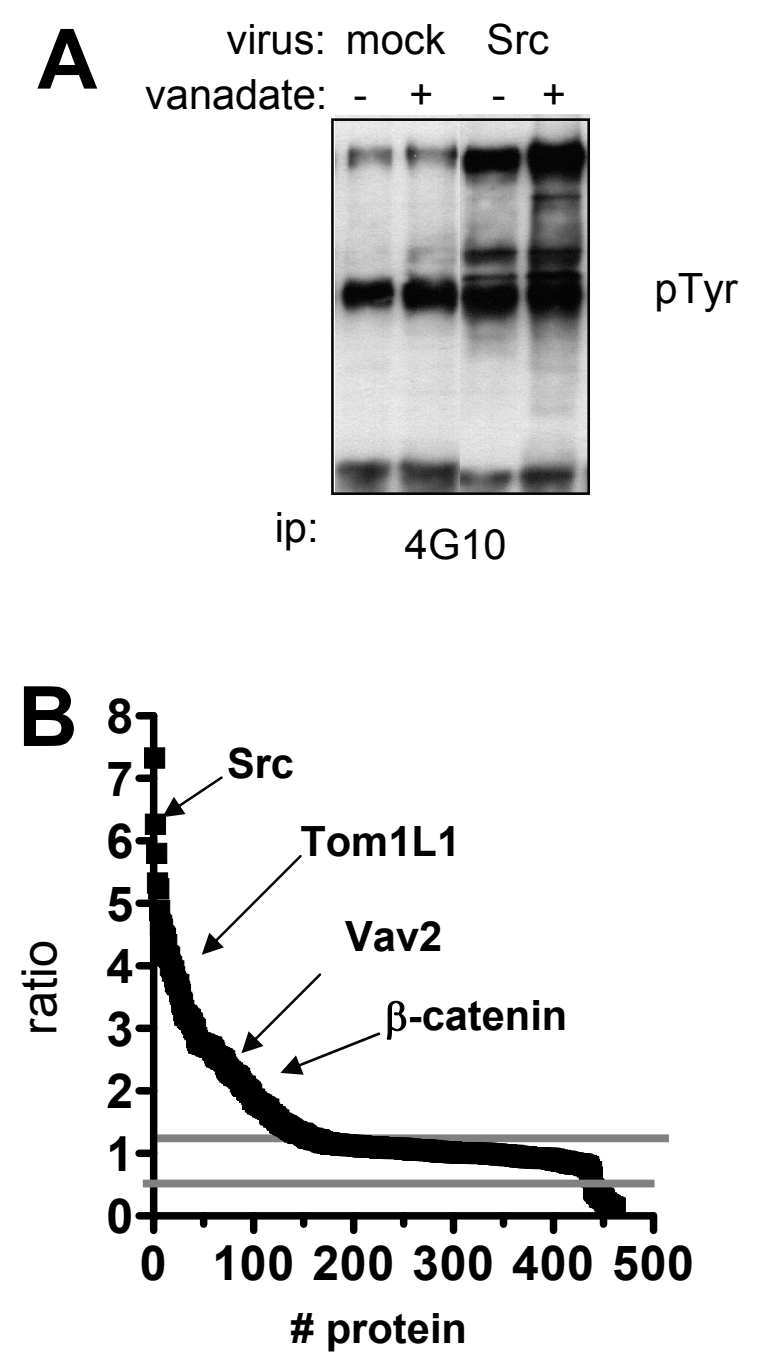
A

virus: mock Src

ip: $\begin{aligned} & \text { aSam68 } \\ & \text { aSam68 }\end{aligned}$

mock Src

mock Src

-pShp2

-Shp2

\begin{tabular}{|l}
\hline$-p V a v 2$ \\
\hline$\square$ avav2
\end{tabular}

ip: $\alpha \mathrm{Cbl}$
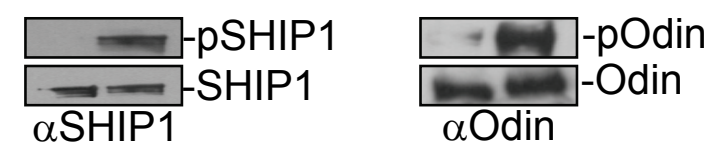

ip: $\alpha \mathrm{PLC} \gamma$
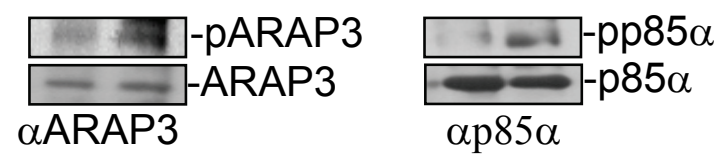

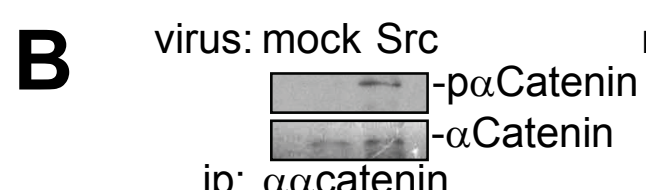

mock Src

mock Src

ip: $\alpha \alpha$ catenin

$-p \beta C$ Catenin
$\alpha \beta$ catenin

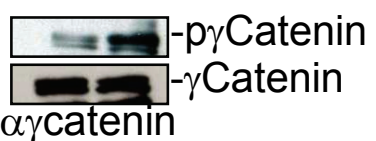

$$
\text { ip: } \frac{\square-p p 120}{\alpha p 120}-p 120
$$
-pRadixin -Radixin

ip: $\overline{\alpha \text { Radixin }}$
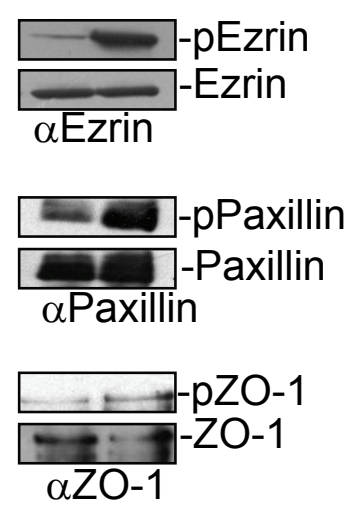

ip: $\alpha$ Calponin

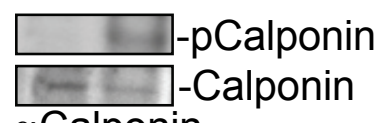
- $-p I R S p 53$ -IRSp53

ip: $\alpha$ IRSp53

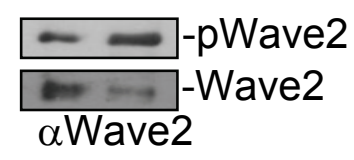

C virus: mock Src

ip: $\alpha \operatorname{Tom} 1 \mathrm{~L} 1$

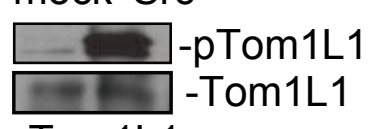

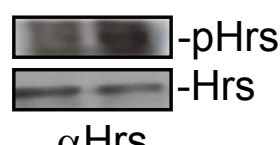

ip: $\alpha \mathrm{Hrs}$ mock Src

\begin{tabular}{l}
\hline -pStam \\
\hline -Stam \\
$\alpha$ Stam2
\end{tabular}

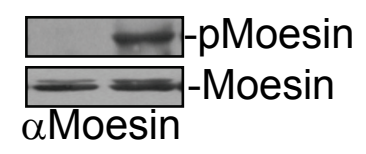

$\square-p p 130$ Cas
$\square-p 130$ Cas

$\alpha \mathrm{Cas}$

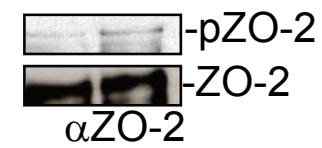

mock Src

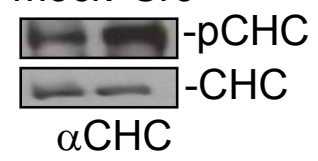




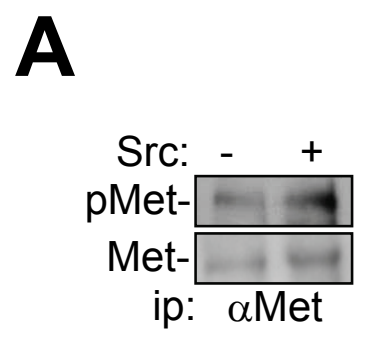

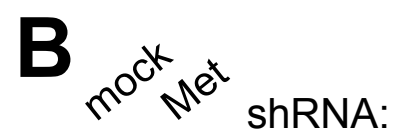
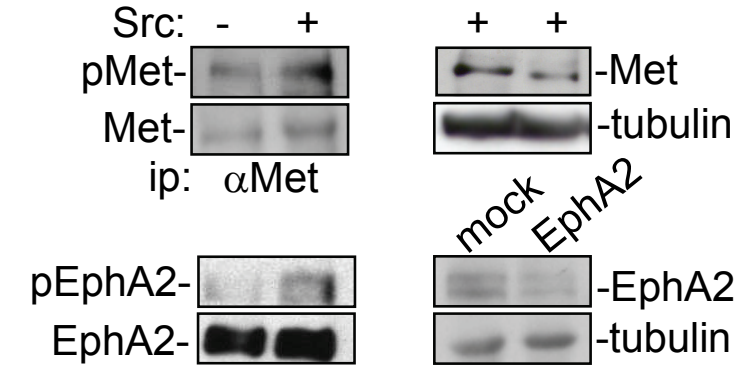

ip: $\alpha$ EphA2
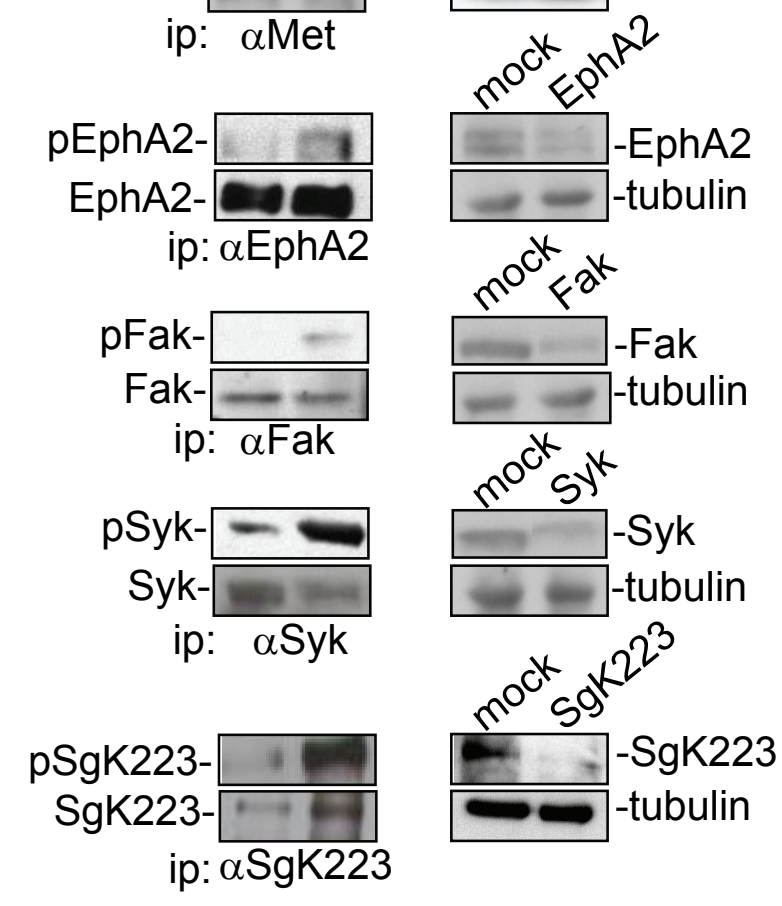

D

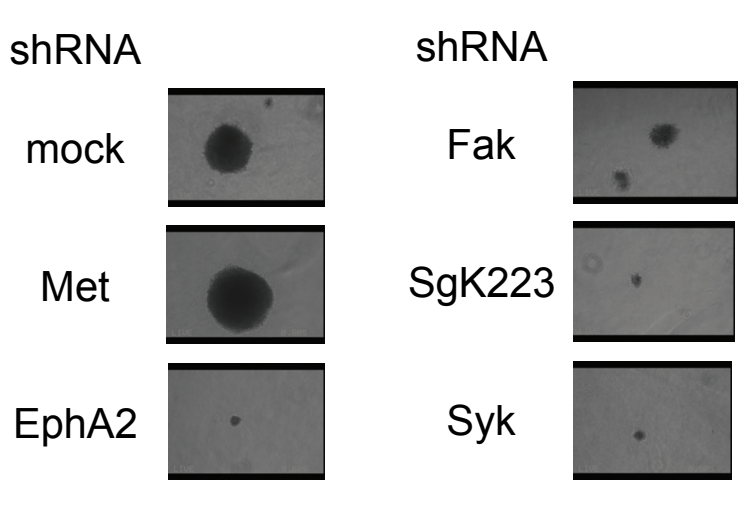

\section{C}

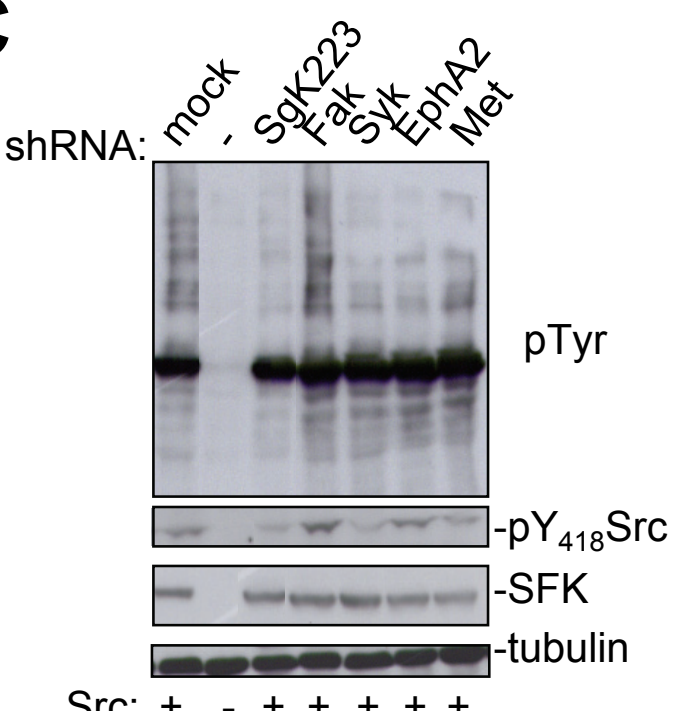

Src: +-+++++

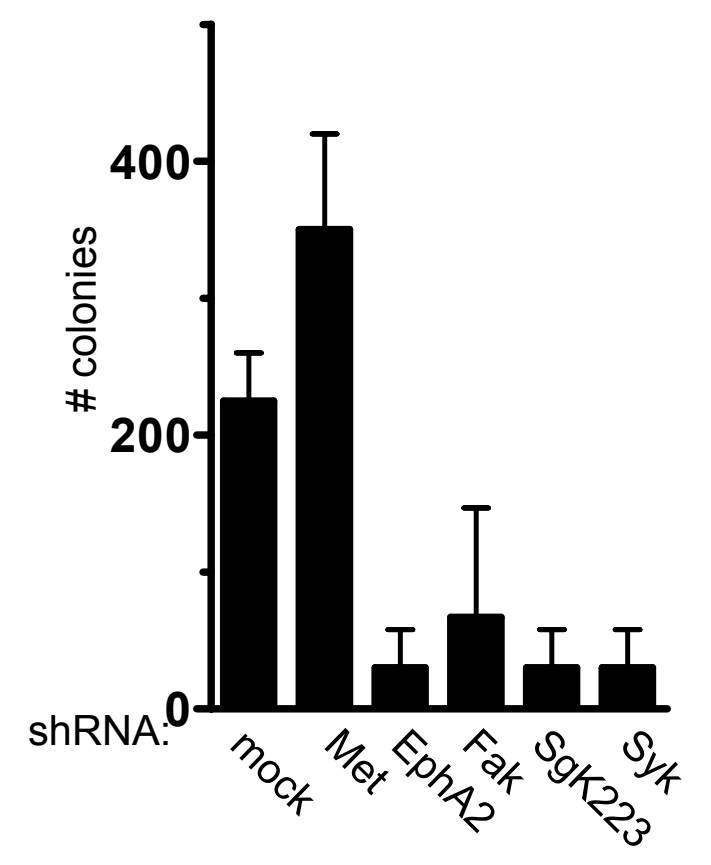




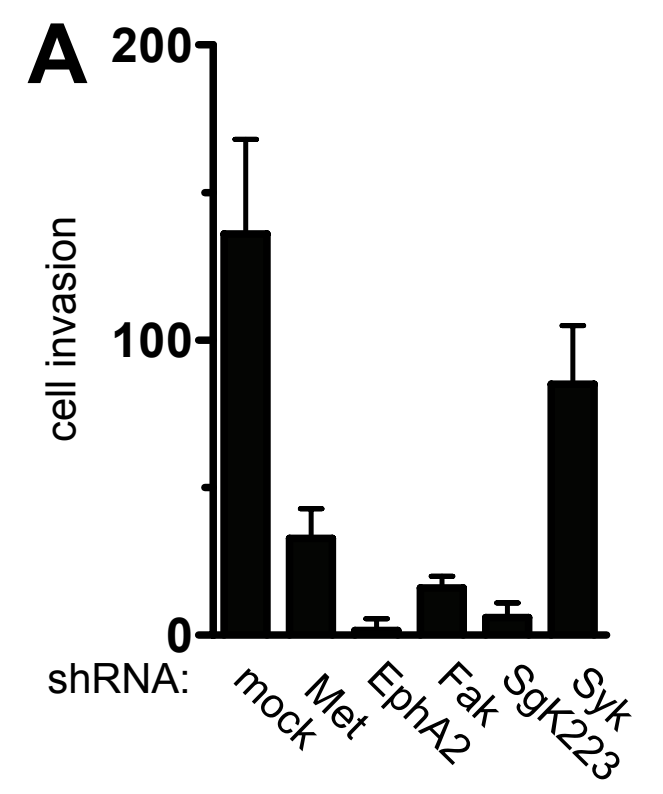

B

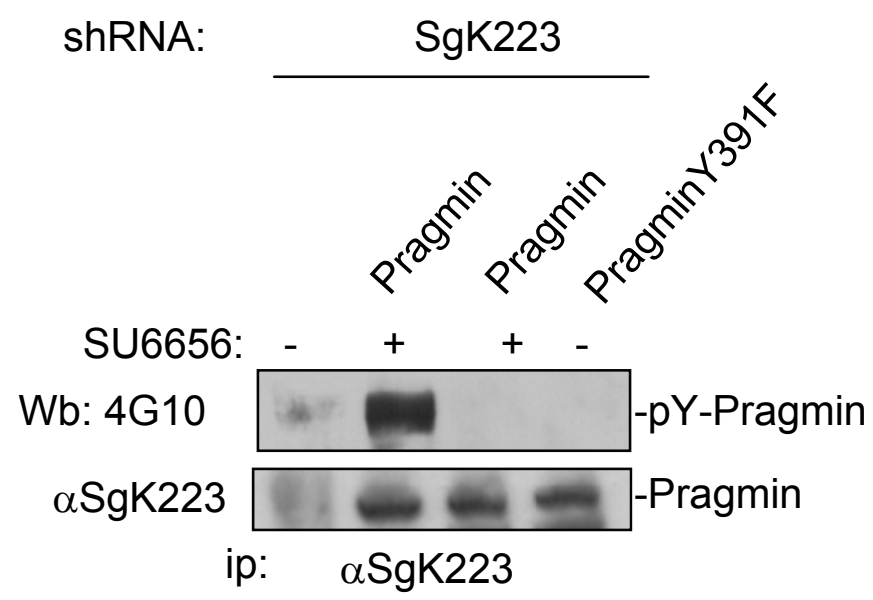

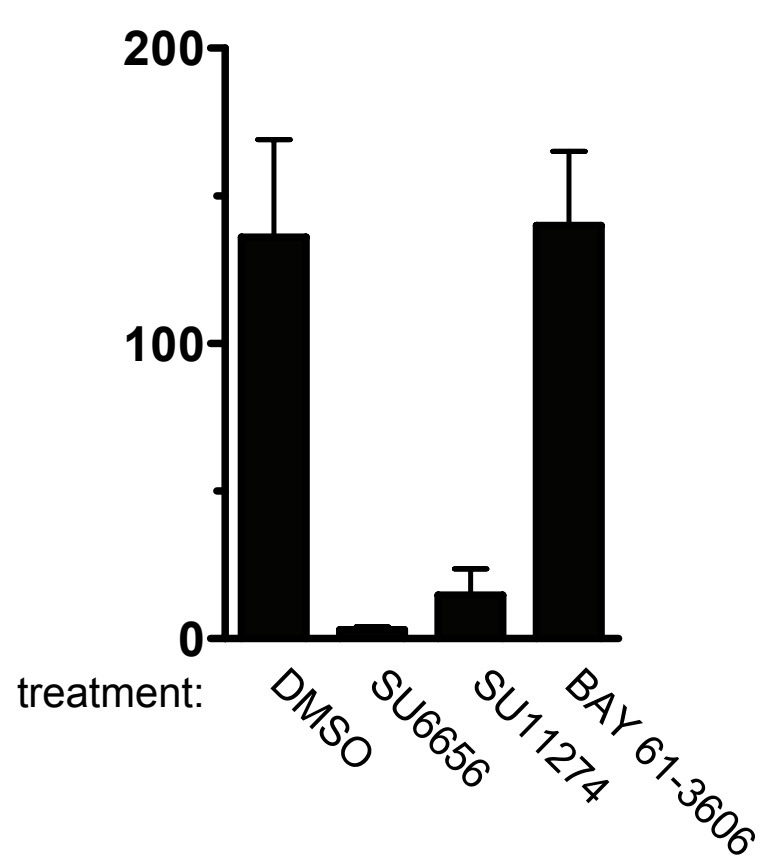

C

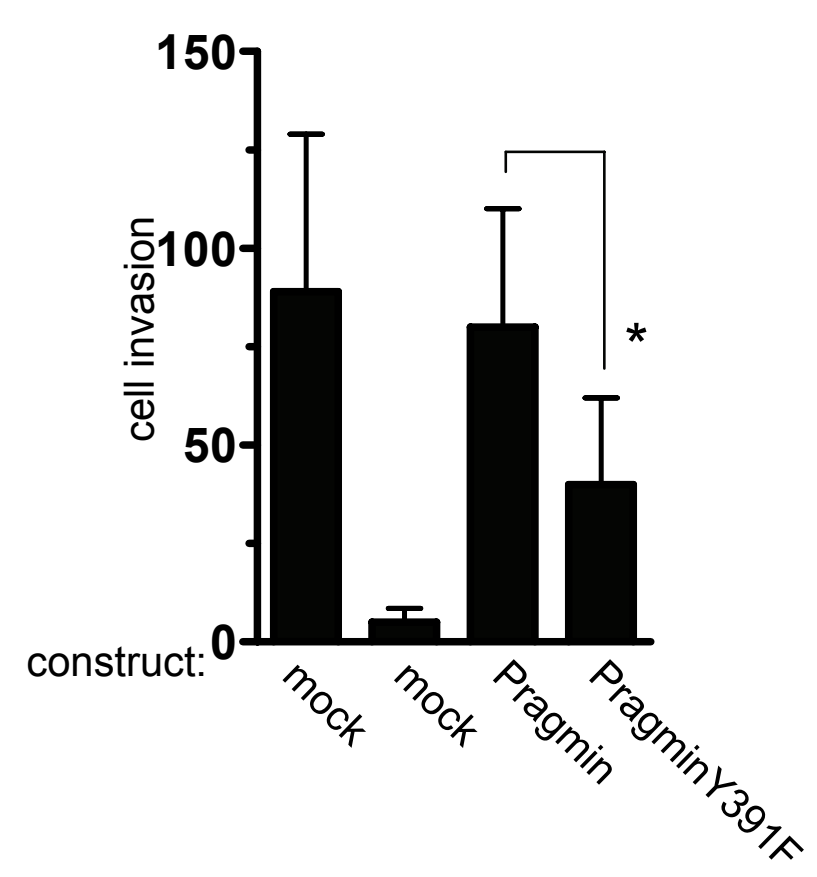


A

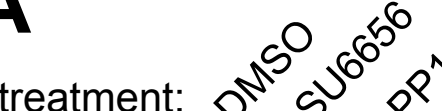
treatment: $Q^{N} \mathrm{~s}^{\mathrm{j}} \mathrm{Q}^{\wedge}$ pEphA2-

EphA2-

ip: $\quad \alpha$ EphA2

pSyk-

Syk-

ip:

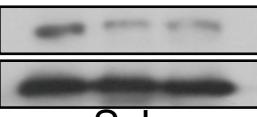

$\alpha$ Syk
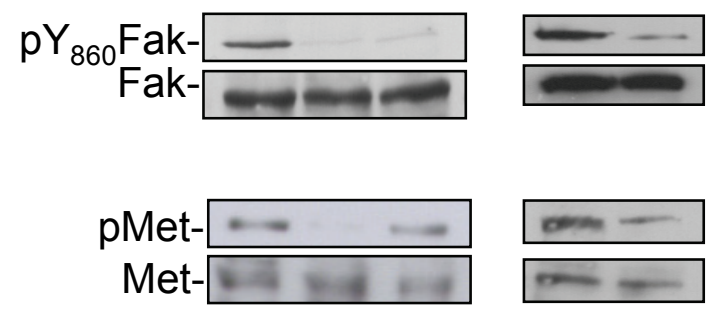

ip: $\quad \alpha M e t$

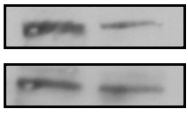

$\alpha$ Met
B
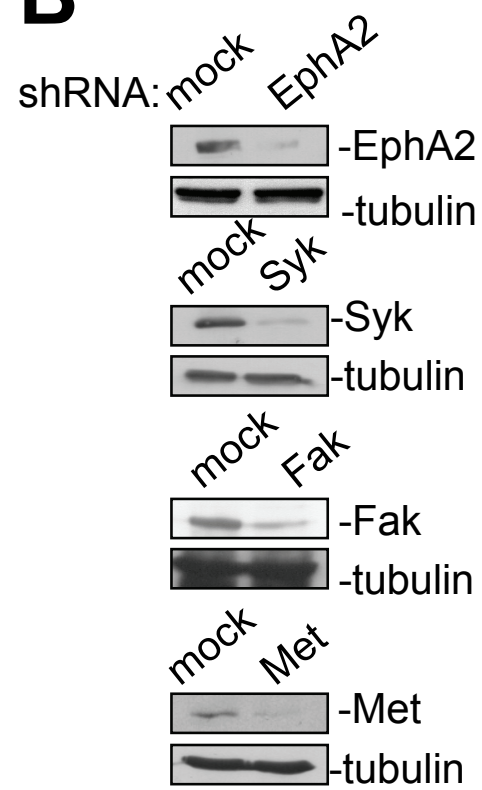

C

SU11274 $(\mu \mathrm{M}): 0 \quad 2 \quad 5$

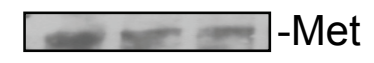

ip: $\quad \alpha$ Met

BAY 61-3606 $(\mu \mathrm{M}): 0 \quad 1 \quad 5 \quad 10$

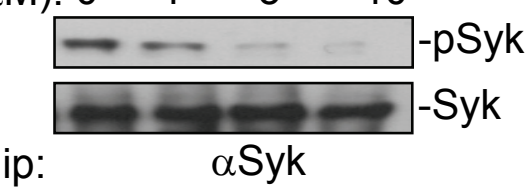

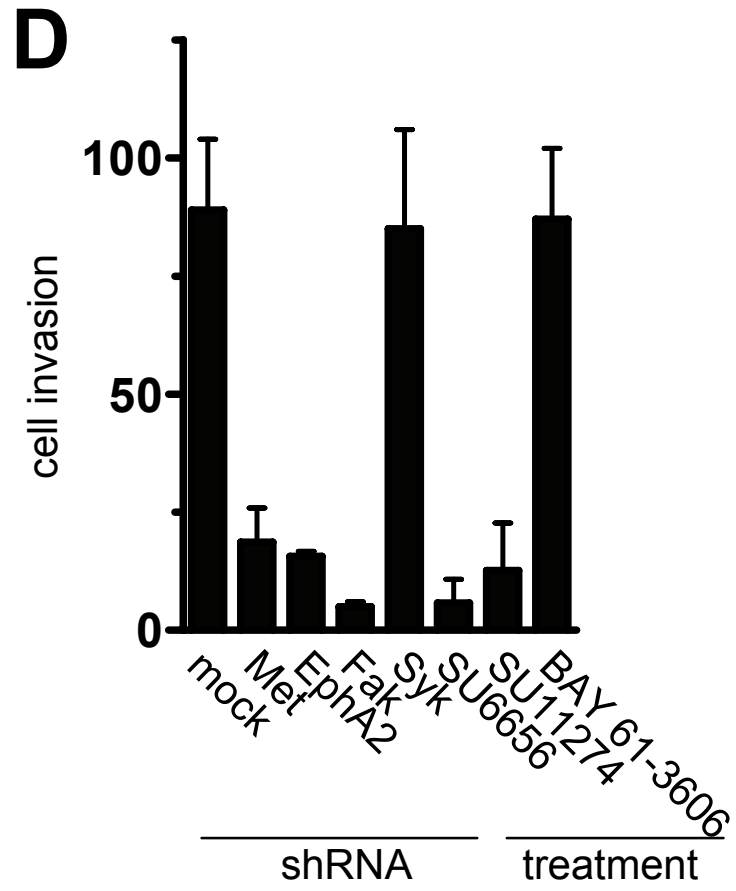

